DOI: 10.17516/1997-1397-2021-14-6-756-767

УДК 517.9

\title{
Cyclic Behavior of Simple Models in Hypoplasticity and Plasticity with Nonlinear Kinematic Hardening
}

\author{
Victor A. Kovtunenko* \\ University of Graz, NAWI Graz \\ Graz, Austria \\ Lavrent'ev Institute of Hydrodynamics SB RAS \\ Novosibirsk, Russian Federation \\ Erich Bauer ${ }^{\dagger}$ \\ Graz University of Technology, \\ Graz, Austria \\ Ján Eliaš \\ University of Graz, NAWI Graz \\ Graz, Austria \\ Pavel Krejčîi ${ }^{\S}$ \\ Czech Technical University in Prague \\ Prague, Czech Republic \\ Giselle A. Monteiro \\ Institute of Mathematics, Czech Academy of Sciences \\ Prague, Czech Republic \\ Lenka Straková (Siváková)\| \\ Czech Technical University in Prague \\ Prague, Czech Republic
}

Received 27.06.2021, received in revised form 10.07.2021, accepted 10.09.2021

\begin{abstract}
The paper gives insights into modeling and well-posedness analysis driven by cyclic behavior of particular rate-independent constitutive equations based on the framework of hypoplasticity and on the elastoplastic concept with nonlinear kinematic hardening. Compared to the classical concept of elastoplasticity, in hypoplasticity there is no need to decompose the deformation into elastic and plastic parts. The two different types of nonlinear approaches show some similarities in the structure of the constitutive relations, which are relevant for describing irreversible material properties. These models exhibit unlimited ratchetting under cyclic loading. In numerical simulation it will be demonstrated, how a shakedown behavior under cyclic loading can be achieved with a slightly enhanced simple hypoplastic equations proposed by Bauer.
\end{abstract}

Keywords: plasticity, hypoplasticity, rate-independent system, hysteresis, cyclic behaviour, modeling, well-posedness, numerical simulation.

Citation: V.A. Kovtunenko, E. Bauer, J. Eliaš, P. Krejčí, G.A. Monteiro, L. Straková (Siváková), Cyclic Behavior of Simple Models in Hypoplasticity and Plasticity with Nonlinear Kinematic Hardening, J. Sib. Fed. Univ. Math. Phys., 2021, 14(6), 756-767. DOI: 10.17516/1997-1397-2021-14-6-756-767.

*victor.kovtunenko@uni-graz.at https://orcid.org/0000-0001-5664-2625

$\dagger$ †erich.bauer@tugraz.at https://orcid.org/0000-0003-2049-5947

¥jan.elias@uni-graz.at https://orcid.org/0000-0002-9768-4124

$\S$ krejci@math.cas.cz https://orcid.org/ 0000-0002-7579-6002

Igam@math.cas.cz https://orcid.org/0000-0001-9651-5719

"lenka.sivak@gmail.com https://orcid.org/ 0000-0001-8839-6676

(C) Siberian Federal University. All rights reserved 


\section{Introduction}

Classical elastoplasticity models based on the concept of yield surface, see, e.g., $[17,18$, $26,28,29,31,32]$, have nice mathematical properties and are therefore often used in solving practical problems involving inelastic rate-independent materials. In particular, when coupling the elastoplastic constitutive law with momentum balance equations in continuum mechanics, the monotonicity and continuity of the constitutive operator of elastoplasticity makes it possible to use conventional methods for constructing the solution.

However, as observed in many experiments, the strain-stress law in real materials exhibits ratchetting, that is, an irreversible progressive shift of the strain-stress path along the strain axis, which cannot be explained within classical elastoplasticity theory. Ratchetting is, however, still a rate-independent phenomenon in contrast to $c r e e p$, which is an effect of rate-dependent viscosity.

An incrementally non-linear constitutive equation of the rate type within the framework of hypoplasticity was introduced by Kolymbas [22]. Based on the general concept of hypoplasticity, particular versions for modeling the behavior of soil and broken rockfill materials have been proposed in the literature on geomechanics. In the present paper, a simplified version of the hypoplastic model originally developed for cohesionless granular materials proposed by [3] and [19] will be considered. For other variational approaches to modelling of granular and plastic media we cite $[1,21,34]$.

Hypoplasticity offers a natural tool for modeling ratchetting. On the other hand, one drawback of hypoplasticity is the lack of continuity with respect to the sup-norm. In other words, small inaccuracies of the input data may produce after a large number of cycles large inaccuracies at the output. This is the reason why during the last decades, many modifications and refinements have been developed in order to combine the ideas of hypoplasticity and elastoplasticity to exploit the advantages of both models, for example $[2,9,14-16,30]$.

The structure of the present contribution is the following one. In Section 1. the classical elastoplastic concept is introduced, and a modification of hardening equations due to ArmstrongFrederick, Bower, and Chaboche is provided with respect to local and global well-posedness. In Section 2. mathematical modeling of hypoplastic constitutive relations is outlined and supported by existence theorems for adopted simplifications by the rate and the Cauchy problems. In particular, further we rely on simplified hypoplastic equations proposed by Bauer. Finally, in Section 3. we present an analytical example for coaxial and homogeneous deformation under isotopic stress cycles. We present our findings during numerical simulation of the shakedown behavior that overcome the drawback of unlimited ratchetting phenomenon.

\section{Kinematic hardening models}

The classical Prandtl-Reuss theory of elastoplasticity (e.g., [26, 32]) consists in assuming additive decomposition of the strain tensor $\varepsilon=\left(\varepsilon_{i j}\right)_{i, j=1}^{3}$ into the elastic component $\varepsilon^{\mathrm{e}}$ and plastic component $\varepsilon^{\mathrm{p}}$, that is,

$$
\varepsilon=\varepsilon^{\mathrm{e}}+\varepsilon^{\mathrm{p}} .
$$

Melan [28] and Prager [31] extended the theory by assuming that also the stress tensor $\boldsymbol{\sigma}=$ $=\left(\sigma_{i j}\right)_{i, j=1}^{3}$ is decomposed into a plastic component $\boldsymbol{\sigma}^{\mathrm{p}}$ and the so-called backstress $\boldsymbol{\sigma}^{\mathrm{b}}$ in the form

$$
\sigma=\sigma^{\mathrm{p}}+\sigma^{\mathrm{b}} .
$$

A linear elasticity law is assumed between $\varepsilon^{\mathrm{e}}$ and $\sigma$. More specifically, a symmetric positive definite tensor $\mathbf{A}=\left(A_{i j k l}\right)_{i, j, k, l=1}^{3}$ is given such that

$$
\sigma=\mathbf{A} \varepsilon^{\mathrm{e}} .
$$


The values of the plastic stress $\boldsymbol{\sigma}^{\mathrm{p}}$ are restricted to a domain of admissible stresses $\mathcal{Z} \subset \mathbb{R}_{\text {sym }}^{3 \times 3}$ in the space $\mathbb{R}_{\mathrm{sym}}^{3 \times 3}$ of symmetric $3 \times 3$ tensors. As soon as $\boldsymbol{\sigma}^{\mathrm{p}}$ reaches the boundary of $\mathcal{Z}$, plastic yielding occurs. Here, we assume the von Mises yield criterion stated in terms of the deviator $\left(\boldsymbol{\sigma}^{\mathrm{p}}\right)^{*}=\boldsymbol{\sigma}^{\mathrm{p}}-\operatorname{tr}\left(\boldsymbol{\sigma}^{\mathrm{p}}\right) \mathbf{I} / 3$ of the plastic strain tensor

$$
\left(\boldsymbol{\sigma}^{\mathrm{p}}\right)^{*}: \boldsymbol{\sigma}^{\mathrm{p}} \leqslant r
$$

where the double-dot implies the scalar product of second-order tensors, $\mathbf{I}$ is the identity tensor, and $r>0$ is given constant. In other words, $\mathcal{Z}$ is a cylinder in the space of symmetric $3 \times 3$ tensors along the I-axis.

In variational form, the yield condition is stated as

$$
\dot{\varepsilon}^{\mathrm{p}}:\left(\boldsymbol{\sigma}^{\mathrm{p}}-\mathbf{z}\right) \geqslant 0 \quad \forall \mathbf{z} \in \mathcal{Z} .
$$

Geometrically, it means that the plastic strain rate $\dot{\boldsymbol{\varepsilon}}^{\mathrm{p}}$ points in the outward normal direction to the boundary of $\mathcal{Z}$ if $\boldsymbol{\sigma}^{\mathrm{p}}$ is on the boundary, and vanishes if $\boldsymbol{\sigma}^{\mathrm{p}}$ is in the interior of $\mathcal{Z}$. This is also called the normality rule in the literature.

The constitutive equation for the backstress $\boldsymbol{\sigma}^{\mathrm{b}}$ in (2) accounts for kinematic hardening. The Melan-Prager setting consists in choosing a linear relation between $\sigma^{\mathrm{b}}$ and $\varepsilon^{\mathrm{p}}$ in the form

$$
\boldsymbol{\sigma}^{\mathrm{b}}=C \varepsilon^{\mathrm{p}}
$$

where the hardening parameter $C>0$ is a constant, while $C=0$ would correspond to the original Prandtl-Reuss model. It is well known (see, e.g., $[17,18,26,29]$ ) that for $C>0$, the system of equations (1)-(6) defines well-posed constitutive operators in both the stress-controlled case $\boldsymbol{\varepsilon}=\mathbf{F}_{\mathrm{MP}}(\boldsymbol{\sigma})$ and strain-controlled case $\boldsymbol{\sigma}=\mathbf{G}_{\mathrm{MP}}(\varepsilon)$ with operators $\mathbf{F}_{\mathrm{MP}}$ and $\mathbf{G}_{\mathrm{MP}}$ acting in the space of absolutely continuous functions with values in $\mathbb{R}_{\mathrm{sym}}^{3 \times 3}$. The subscript "MP" stands for Melan-Prager, indeed.

The ratchetting effect produced by the linear relation (6) is very weak. This is why Armstrong and Frederick proposed in [2] a nonlinear "hypoplastic" modification of the hardening equation (6) in the form

$$
\dot{\boldsymbol{\sigma}}^{\mathrm{b}}=\gamma\left(R \dot{\varepsilon}^{\mathrm{p}}-\boldsymbol{\sigma}^{\mathrm{b}}\left\|\dot{\varepsilon}^{\mathrm{p}}\right\|\right)
$$

where $\gamma>0, R>0$ are given constants, and $\|\cdot\|$ is the Frobenius norm

$$
\left\|\dot{\varepsilon}^{\mathrm{p}}\right\|=\left(\dot{\varepsilon}^{\mathrm{p}}: \dot{\varepsilon}^{\mathrm{p}}\right)^{1 / 2} .
$$

In order to improve the description of ratchetting effects which occur during the elastoplastic deformation of railway rails, Bower in [9] further refined the Armstrong-Frederick model by introducing a second backstress component $\dot{\boldsymbol{\sigma}}^{\beta}$ satisfying the constitutive equations

$$
\begin{aligned}
& \dot{\boldsymbol{\sigma}}^{\mathrm{b}}=\gamma\left(R \dot{\boldsymbol{\varepsilon}}^{\mathrm{p}}-\left(\boldsymbol{\sigma}^{\mathrm{b}}-\boldsymbol{\sigma}^{\beta}\right)\left\|\dot{\boldsymbol{\varepsilon}}^{\mathrm{p}}\right\|\right), \\
& \dot{\boldsymbol{\sigma}}^{\beta}=c\left(\boldsymbol{\sigma}^{\mathrm{b}}-\boldsymbol{\sigma}^{\beta}\right)\left\|\dot{\boldsymbol{\varepsilon}}^{\mathrm{p}}\right\|,
\end{aligned}
$$

where $c>0$ is an additional constant.

Another modification of the Armstrong-Frederick kinematic hardening law (7) was proposed by Chaboche in a series of papers [14-16], and consists in representing the backstress $\boldsymbol{\sigma}^{\mathrm{b}}$ as a sum

$$
\boldsymbol{\sigma}^{\mathrm{b}}=\sum_{k \in J} \boldsymbol{\sigma}_{k}^{\mathrm{b}}
$$

of partial backstresses $\boldsymbol{\sigma}_{k}^{\mathrm{b}}$ over an index set $J$, each of them satisfying the Armstrong-Frederick kinematic hardening condition

$$
\dot{\boldsymbol{\sigma}}_{k}^{\mathrm{b}}=\gamma(k)\left(R(k) \dot{\boldsymbol{\varepsilon}}^{\mathrm{p}}-\boldsymbol{\sigma}_{k}^{\mathrm{b}}\left\|\dot{\boldsymbol{\varepsilon}}^{\mathrm{p}}\right\|\right)
$$


for $k \in J$ with constants $\gamma(k)>0, R(k)>0$.

All the stress and strain controlled constitutive operators $\mathbf{F}_{\mathrm{AF}}, \mathbf{G}_{\mathrm{AF}}, \mathbf{F}_{\mathrm{B}}, \mathbf{G}_{\mathrm{B}}, \mathbf{F}_{\mathrm{Ch}}, \mathbf{G}_{\mathrm{Ch}}$ generated by the Armstrong-Frederick, Bower, and Chaboche models can be shown to be well-posed in spaces $W^{1,1}\left(0, T ; \mathbb{R}_{\mathrm{sym}}^{3 \times 3}\right)$ of tensor-valued absolutely continuous functions, as well as in the space $C B V\left(0, T ; \mathbb{R}_{\mathrm{sym}}^{3 \times 3}\right)$ of continuous functions with bounded variation. The following results are proved in $[10-12]$.

Proposition 1 (Local well-posedness). If the initial conditions satisfy a natural compatibility condition, then the operator $\mathbf{F}_{\mathrm{AF}}$ of the stress controlled Armstrong-Frederick model is well defined on the domain

$$
\mathcal{D}_{\mathrm{FAF}}=\left\{\boldsymbol{\sigma} \in C B V\left(0, T ; \mathbb{R}_{\mathrm{sym}}^{3 \times 3}\right): \quad\left\|(\boldsymbol{\sigma})^{*}\right\|_{\infty}<R+r\right\},
$$

and it is continuous with respect to the sup-norm and Lipschitz continuous with respect to the $W^{1,1}$-norm on every subset of input functions with uniformly bounded variation.

Proposition 2 (Global well-posedness). Assuming that the initial data satisfy natural compatibility conditions and under suitable technical hypotheses, the constitutive operators $\mathbf{F}_{\mathrm{B}}, \mathbf{G}_{\mathrm{B}}, \mathbf{F}_{\mathrm{Ch}}, \mathbf{G}_{\mathrm{Ch}}, \mathbf{G}_{\mathrm{AF}}$ for the stress and strain controlled Bower and Chaboche models as well as for the strain controlled Armstrong-Frederick model are well defined and possess the continuity properties as in Proposition 1 without any restriction on the size of the input functions.

\section{Initial boundary value problems in hypoplasticity}

In the space $\mathbb{R}_{\mathrm{sym}}^{3 \times 3}$ of second-order symmetric $3 \times 3$ tensors, the nonlinear theory of rateindependent materials is constituted by response between the Cauchy stress $\boldsymbol{\sigma}=\left(\sigma_{i j}\right)_{i, j=1}^{3}$, its objective rate $\stackrel{\circ}{\sigma}=\left(\stackrel{\circ}{\sigma}_{i j}\right)_{i, j=1}^{3}$, and the strain rate $\dot{\varepsilon}=\left(\dot{\varepsilon}_{i j}\right)_{i, j=1}^{3}$ given in the general form of an implicit function (see [33]):

$$
\mathbf{f}(\boldsymbol{\sigma}, \stackrel{\circ}{\boldsymbol{\sigma}}, \dot{\varepsilon})=\mathbf{0} .
$$

For the function $\mathbf{f}$ positively homogeneous of degree one with respect to rates:

$$
\mathbf{f}(\boldsymbol{\sigma}, \lambda \stackrel{\circ}{\boldsymbol{\sigma}}, \lambda \dot{\boldsymbol{\varepsilon}})=\lambda \mathbf{f}(\boldsymbol{\sigma}, \stackrel{\circ}{\boldsymbol{\sigma}}, \dot{\boldsymbol{\varepsilon}}) \quad \text { for } \lambda>0,
$$

the constitutive law (13) is rate-independent. The special cases of (13) satisfying (14) is the hypoelastic law (that cannot be derived from a strain energy, see [35]):

$$
\stackrel{\circ}{\boldsymbol{\sigma}}-\mathbf{L}(\boldsymbol{\sigma}): \dot{\boldsymbol{\varepsilon}}=\mathbf{0}, \quad \mathbf{L}=\left(L_{i j k l}\right)_{i, j, k, l=1}^{3},
$$

which is linear with respect to both stress and strain rates. Here the colon stands for the double contraction of the fourth-order tensor $\mathbf{L}(\boldsymbol{\sigma})$ with the second-order tensor $\dot{\varepsilon}$. To extend (15) for an inelastic behavior such that $\mathbf{f}(\boldsymbol{\sigma}, \stackrel{\circ}{\boldsymbol{\sigma}},-\dot{\varepsilon}) \neq-\mathbf{f}(\boldsymbol{\sigma}, \stackrel{\circ}{\boldsymbol{\sigma}}, \dot{\varepsilon})$, the nonlinearity of function $\mathbf{f}$ in $\dot{\varepsilon}$ can, for instance, be expressed as

$$
\stackrel{\circ}{\sigma}-\mathbf{L}(\boldsymbol{\sigma}): \dot{\varepsilon}-\mathbf{N}(\boldsymbol{\sigma})\|\dot{\varepsilon}\|=\mathbf{0},
$$

where the tensor $\mathbf{N}=\left(N_{i j}\right)_{i, j=1}^{3}$, the Frobenius norm $\|\dot{\varepsilon}\|^{2}=\operatorname{tr}\left(\dot{\boldsymbol{\varepsilon}}^{2}\right)$, and $\operatorname{tr}(\cdot)$ stands for the trace. Function (16) also satisfies (14) and represents the concept of hypoplasticity introduces by Kolymbas [22]. As a consequence of the nonlinearity in the strain rate function (16) is apt to model hysteresis behavior. Particular constitutive relations based on (16) were developed for describing the mechanical behavior of granular materials and soils in geomechanics (e.g. $[8,20,23,27])$. 
The constitutive equation (16) is completed with the equilibrium equation

$$
-\operatorname{div} \boldsymbol{\sigma}=\mathbf{F}
$$

under the given body force $\mathbf{F} \in \mathbb{R}^{3}$, and the symmetric part of the velocity gradient

$$
\dot{\varepsilon}=\frac{1}{2}\left(\nabla \mathbf{v}+\nabla \mathbf{v}^{\top}\right)
$$

with the velocity vector $\mathbf{v} \in \mathbb{R}^{3}$. The system (16)-(18) for unknown $\boldsymbol{\sigma}$ and $\mathbf{v}$ is supported by usual boundary and initial conditions. An appropriate objective stress rate for the present hypoplastic constitutive equation is discussed in [4]. In the following we restrict ourselves to the time derivative, e.g. for purely coaxial deformations, which implies that

$$
\stackrel{\circ}{\sigma}=\dot{\sigma} .
$$

Moreover, for cohesionless granular materials only negative principal stresses (which are three eigenvalues of the stress tensor) are relevant:

$$
\sigma_{1}<0, \quad \sigma_{2}<0, \quad \sigma_{3}<0,
$$

wherefrom it follows that the first invariant of the stress tensor is negative

$$
\operatorname{tr}(\boldsymbol{\sigma})=\sigma_{1}+\sigma_{2}+\sigma_{3}<0 .
$$

In general, mathematical analysis of highly nonlinear governing relations (16)-(20) is fully open and relies on investigation of simplified models as follows.

In one approach, a suitable stress field $\boldsymbol{\sigma}$ satisfying (17) and (20) is prescribed a-priori. Formally, replacing the objective derivative by the time derivative (19), we differentiate the equilibrium equation (17) with respect to time and substitute (16) there. As the result, it follows the rate problem for unknown velocity $\mathbf{v}$ :

$$
-\operatorname{div}(\mathbf{L}(\boldsymbol{\sigma}): \dot{\varepsilon}+\mathbf{N}(\boldsymbol{\sigma})\|\dot{\varepsilon}\|)=\dot{\mathbf{F}}
$$

by means of the strain velocity from (18) that is reminded here for completeness

$$
\dot{\varepsilon}=\frac{1}{2}\left(\nabla \mathbf{v}+\nabla \mathbf{v}^{\top}\right),
$$

and supported by usual boundary conditions of Dirichlet and Neumann type. The rate problem (22) is well-known as the CloE model (see [13]). Applying the Browder-Minty existence theorem, we can straightforwardly formulate the following result.

Proposition 3 (Well-posedness for rate problem). Let the operator of (22), (23)

$$
\mathbf{v} \mapsto-\operatorname{div}\left(\mathbf{L}(\boldsymbol{\sigma}): \frac{1}{2}\left(\nabla \mathbf{v}+\nabla \mathbf{v}^{\top}\right)+\mathbf{N}(\boldsymbol{\sigma}) \frac{1}{2}\left\|\nabla \mathbf{v}+\nabla \mathbf{v}^{\top}\right\|\right) \quad \text { for } \mathbf{v} \in H_{\mathrm{loc}}^{1}\left(\mathbb{R}_{\mathrm{sym}}^{3 \times 3}\right)
$$

be bounded, continuous, coercive, and monotone for some fixed $\boldsymbol{\sigma}$. Then the operator is surjective, and the rate problem has at least one solution.

In another approach, a suitable strain rate $\dot{\varepsilon}$ satisfying (18) is prescribed a-priori. In this case, considering the constitutive equation (16) in isolation, we get the Cauchy problem for unknown stress $\sigma \in \mathbb{R}_{\mathrm{sym}}^{3 \times 3}$ :

$$
\stackrel{\circ}{\sigma}=\mathbf{L}(\boldsymbol{\sigma}): \dot{\varepsilon}+\mathbf{N}(\boldsymbol{\sigma})\|\dot{\varepsilon}\|
$$


under the initial condition

$$
\boldsymbol{\sigma}(0)=\boldsymbol{\sigma}^{0}
$$

and subject to the constraint of negative principal stresses (20). Applying the simplifying assumption (19), the existence of solution to the corresponding nonlinear ODE system

$$
\frac{d \boldsymbol{\sigma}}{d t}=\mathbf{L}(\boldsymbol{\sigma}): \dot{\varepsilon}+\mathbf{N}(\boldsymbol{\sigma})\|\dot{\varepsilon}\|
$$

follows from the Cauchy-Lipschitz theorem, which provides the following result.

Proposition 4 (Global well-posedness for Cauchy problem). For fixed $\dot{\varepsilon}$ let the operator

$$
\boldsymbol{\sigma} \mapsto \mathbf{L}(\boldsymbol{\sigma}): \dot{\boldsymbol{\varepsilon}}+\mathbf{N}(\boldsymbol{\sigma})\|\dot{\boldsymbol{\varepsilon}}\| \quad \text { for } \boldsymbol{\sigma} \in \mathbb{R}_{\mathrm{sym}}^{3 \times 3}
$$

be Lipschitz-continuous. Then the Cauchy problem (26), (27) has a global in time solution $\boldsymbol{\sigma}(t)$.

However, Proposition 4 does not guarantee the negativeness of principal stresses (20).

For example, we consider a simplified version of the hypoplastic model proposed by Bauer [5]

$$
\mathbf{L}(\boldsymbol{\sigma}): \dot{\boldsymbol{\varepsilon}}=c\left(a^{2} \operatorname{tr}(\boldsymbol{\sigma}) \dot{\varepsilon}+\frac{\boldsymbol{\sigma}: \dot{\boldsymbol{\varepsilon}}}{\operatorname{tr}(\boldsymbol{\sigma})} \boldsymbol{\sigma}\right), \quad \mathbf{N}(\boldsymbol{\sigma})=c f_{\mathrm{c}} a\left(2 \boldsymbol{\sigma}-\frac{1}{3} \operatorname{tr}(\boldsymbol{\sigma}) \mathbf{I}\right),
$$

where the colon denotes the double contraction of second-order tensors, I stands for the identity tensor, $c<0$ and $f_{\mathrm{c}}>0$ are scaling parameters. Constant $a>0$ determines the opening of the conical surface, which bounds the admissible stress states in the space of negative principal stress. It can be checked that the nonlinear operator induced by (29) satisfies the assumptions of Proposition 3. However, there is no a priori evidence that $\operatorname{tr}(\boldsymbol{\sigma})$ remains bounded away from zero independently of the input histories $\dot{\varepsilon}$. We cannot therefore guarantee in general the validity of the global Lipschitz continuity condition in Proposition 4 for the case (29), and a detailed local in time analysis will be necessary.

Previously we studied the Bauer model (29) in the context of the nonlinear ODE (27), i.e.

$$
\frac{d \boldsymbol{\sigma}}{d t}=c\left(a^{2} \operatorname{tr}(\boldsymbol{\sigma}) \dot{\varepsilon}+\frac{\boldsymbol{\sigma}: \dot{\boldsymbol{\varepsilon}}}{\operatorname{tr}(\boldsymbol{\sigma})} \boldsymbol{\sigma}+f_{\mathrm{c}} a\left(2 \boldsymbol{\sigma}-\frac{1}{3} \operatorname{tr}(\boldsymbol{\sigma}) \mathbf{I}\right)\|\dot{\boldsymbol{\varepsilon}}\|\right) .
$$

For constant $\dot{\boldsymbol{\varepsilon}}$ we derived an analytical solution to (30) in the closed form, as described in details in [7] (there $f_{\mathrm{c}}=1$ was set). The explicit solution was used to establish asymptotic behavior for the stress under proportional loading (known as Goldscheider's rule) in [7], to prove the Lyapunov stability for the dynamic system in [24], and to outline a feasible region where principal stresses are non-positive in [25]. The solution procedure was extended further to a modified model in [6].

In the following section we construct an explicit solution to (30) for numerical simulation of ratchetting under loading-unloading cycles.

\section{Numerical simulation of ratchetting}

For coaxial and homogeneous deformation the matrix representation of $\boldsymbol{\sigma}$ and $\dot{\varepsilon}$ is as follows:

$$
\boldsymbol{\sigma}=\left[\begin{array}{ccc}
\sigma_{1} & 0 & 0 \\
0 & \sigma_{2} & 0 \\
0 & 0 & \sigma_{3}
\end{array}\right] \quad \text { and } \quad \dot{\varepsilon}=\left[\begin{array}{ccc}
\dot{\varepsilon}_{1} & 0 & 0 \\
0 & \dot{\varepsilon}_{2} & 0 \\
0 & 0 & \dot{\varepsilon}_{3}
\end{array}\right]
$$

Moreover, we consider in the following isotopic compression and isotropic extension starting from an isotropic stress state. Then $\sigma_{1}=\sigma_{2}=\sigma_{3}$ and $\dot{\varepsilon}_{1}=\dot{\varepsilon}_{2}=\dot{\varepsilon}_{3}$ holds. By omitting indices from the notation (hence, we write $\sigma_{i}=\sigma$ and $\dot{\varepsilon}_{i}=\dot{\varepsilon}$ for $i=1,2,3$ ), it follows

$$
\operatorname{tr}(\boldsymbol{\sigma})=3 \sigma, \quad \boldsymbol{\sigma}: \dot{\varepsilon}=3 \sigma \dot{\varepsilon}, \quad\|\dot{\varepsilon}\|=\sqrt{3}|\dot{\varepsilon}| .
$$


Thus, by replacing $d \sigma / d t$ with $\dot{\sigma}$ we obtain from (30) the equation for the principal stress

$$
\dot{\sigma}=3 c \sigma\left(a^{2} \dot{\varepsilon}+\frac{1}{3} \dot{\varepsilon}+f_{\mathrm{c}} \frac{a}{\sqrt{3}}|\dot{\varepsilon}|\right) .
$$

Herein $\dot{\varepsilon}<0$ for the compression (loading) phase and $\dot{\varepsilon}>0$ for the extension (unloading) phase. Hence we obtain two equations

$$
\begin{array}{ll}
\dot{\sigma}=3 c\left(a^{2}+\frac{1}{3}-f_{\mathrm{c}} \frac{a}{\sqrt{3}}\right) \sigma \dot{\varepsilon} & \text { for loading, } \\
\dot{\sigma}=3 c\left(a^{2}+\frac{1}{3}+f_{\mathrm{c}} \frac{a}{\sqrt{3}}\right) \sigma \dot{\varepsilon} & \text { for unloading. }
\end{array}
$$

The equations in (32) and (33) are solved on closed intervals depending on the loading, resp. unloading phase of the cycle. The final state after one loading (resp. unloading) phase becomes an initial state for the following unloading (resp. loading) phase. For the initial loading we assume that $\sigma(0)=\sigma_{0}<0$ and $\varepsilon(0)=0$. The phase switching happens whenever $\sigma$ reaches the prescribed values $\sigma_{I}$ and $\sigma_{I I}$. We assume without loss of generality that $\sigma_{I}<\sigma_{I I}<0$; unloading goes from $\sigma_{I}$ to $\sigma_{I I}$ whilst loading from $\sigma_{I I}$ to $\sigma_{I}$.

Let us denote

$$
\lambda_{ \pm}=3 c\left(a^{2}+\frac{1}{3} \pm f_{\mathrm{c}} \frac{a}{\sqrt{3}}\right) .
$$

Then we can solve the equation $\dot{\sigma}=\lambda_{ \pm} \sigma \dot{\varepsilon}$ on an arbitrary interval $[\tau, T], 0 \leqslant \tau<T$, easily by using the method of separation of variables. We obtain

$$
\sigma(t)=\sigma(\tau) e^{\lambda_{ \pm}(\varepsilon(t)-\varepsilon(\tau))} \quad(\tau \leqslant t \leqslant T) .
$$

We can rewrite the formula (34) above in order to get an equation for the strain $\varepsilon(t)$ for $t \in[\tau, T]$. In particular, the strain $\varepsilon$ at the time $t=T$ reads

$$
\varepsilon(T)=\frac{1}{\lambda_{ \pm}} \ln \left(\frac{\sigma(T)}{\sigma(\tau)}\right)+\varepsilon(\tau) .
$$

Here, $\tau$ is the time at which the un/loading phase of a cycle starts and $T$ is the time at which this phase ends.

In order to do numerical simulations, we need an additional information either on the stress or on the strain. Let us assume that $\dot{\varepsilon}=\operatorname{diag}\left(k_{0}, k_{0}, k_{0}\right)$, where $k_{0} \in \mathbb{R}$. As the constitutive equation (30) describes a rate independent material behavior, the amount of $k_{0}$ is arbitrary. However, the sign of $k_{0}$ defines as to whether the material is subjected to compression, i.e. $\operatorname{sign}\left(k_{0}\right)=-1$, or to extension, i.e. $\operatorname{sign}\left(k_{0}\right)=+1$. Thus, $\varepsilon$ satisfies the equation $\dot{\varepsilon}=k_{0}$ which has an explicit solution, namely,

$$
\varepsilon(t)=k_{0} t+\left(\varepsilon(\tau)-k_{0} \tau\right)
$$

on every interval $[\tau, T]$. By substituting for $\varepsilon(t)$ in (34) we obtain

$$
\sigma(t)=\sigma(\tau) e^{\lambda_{ \pm} k_{0}(t-\tau)} \quad(\tau \leqslant t \leqslant T) .
$$

and, in particular,

$$
T=\frac{1}{\lambda_{ \pm} k_{0}} \ln \left(\frac{\sigma(T)}{\sigma(\tau)}\right)+\tau .
$$

We observe that $\varepsilon(T)>\varepsilon(\tau)$ for every unloading phase $\left(\operatorname{sign}\left(k_{0}\right)=+1\right)$.

The initial loading phase on an interval $\left[\tau_{0}, T_{0}\right]$ starts at $\tau_{0}=0$ with $\sigma(0)=\sigma_{0}$ and $\varepsilon(0)=0$ and ends at the time $T_{0}$, at which $\sigma\left(T_{0}\right)=\sigma_{I}$. Therefore,

$$
T_{0}=\frac{1}{\lambda_{-} k_{0}} \ln \left(\frac{\sigma_{I}}{\sigma_{0}}\right), \quad \sigma(t)=\sigma_{0} e^{\lambda_{-} \varepsilon(t)}=\sigma_{0} e^{\lambda_{-} k_{0} t} \quad \text { and } \quad \varepsilon_{I}:=\varepsilon\left(T_{0}\right)=k_{0} T_{0} .
$$


Then, the first cycle starts with an unloading phase from $\sigma_{I}$ to $\sigma_{I I}$. We solve (33) on $\left[\tau_{1}, T_{1}\right]$ where $\tau_{1}=T_{0}, \sigma\left(\tau_{1}\right)=\sigma_{I}$ and $\varepsilon\left(\tau_{1}\right)=\varepsilon_{I}$. We obtain from (34)-(36) that for $\varepsilon_{I I}:=\varepsilon\left(T_{1}\right)$

$$
T_{1}=\frac{1}{\lambda_{+} k_{0}} \ln \left(\frac{\sigma_{I I}}{\sigma_{I}}\right)+\tau_{1}, \quad \sigma(t)=\sigma_{I} e^{\lambda_{+}\left(\varepsilon(t)-\varepsilon_{I}\right)}=\sigma_{I} e^{\lambda_{+} k_{0}\left(t-\tau_{1}\right)}, \quad \varepsilon_{I I}=k_{0}\left(T_{1}-\tau_{1}\right)+\varepsilon_{I}
$$

since the unloading ends for $\sigma\left(T_{1}\right)=\sigma_{I I}$. Then we switch back to the reloading phase from $\sigma_{I I}$ to $\sigma_{I}$ to complete the first cycle. We solve (32) on yet another interval $\left[\tau_{2}, T_{2}\right]$ with $\tau_{2}=T_{1}$, $\sigma\left(\tau_{2}\right)=\sigma_{I I}$ and $\varepsilon\left(\tau_{2}\right)=\varepsilon_{I I}$. We deduce from (33)-(35) that for $\varepsilon_{I I I}:=\varepsilon\left(T_{2}\right)$

$$
T_{2}=\frac{1}{\lambda_{-} k_{0}} \ln \left(\frac{\sigma_{I}}{\sigma_{I I}}\right)+\tau_{2}, \quad \sigma(t)=\sigma_{I I} e^{\lambda_{-}\left(\varepsilon(t)-\varepsilon_{I I}\right)}=\sigma_{I I} e^{\lambda_{-} k_{0}\left(t-\tau_{2}\right)}, \quad \varepsilon_{I I I}=k_{0}\left(T_{2}-\tau_{2}\right)+\varepsilon_{I I}
$$

since the loading ends for $\sigma\left(T_{2}\right)=\sigma_{I}$. Analogously, we obtain stresses, strains and the respective time intervals for every of the following cycles. A typical response of the material to isotropic stress cycles is shown in Fig. 1.
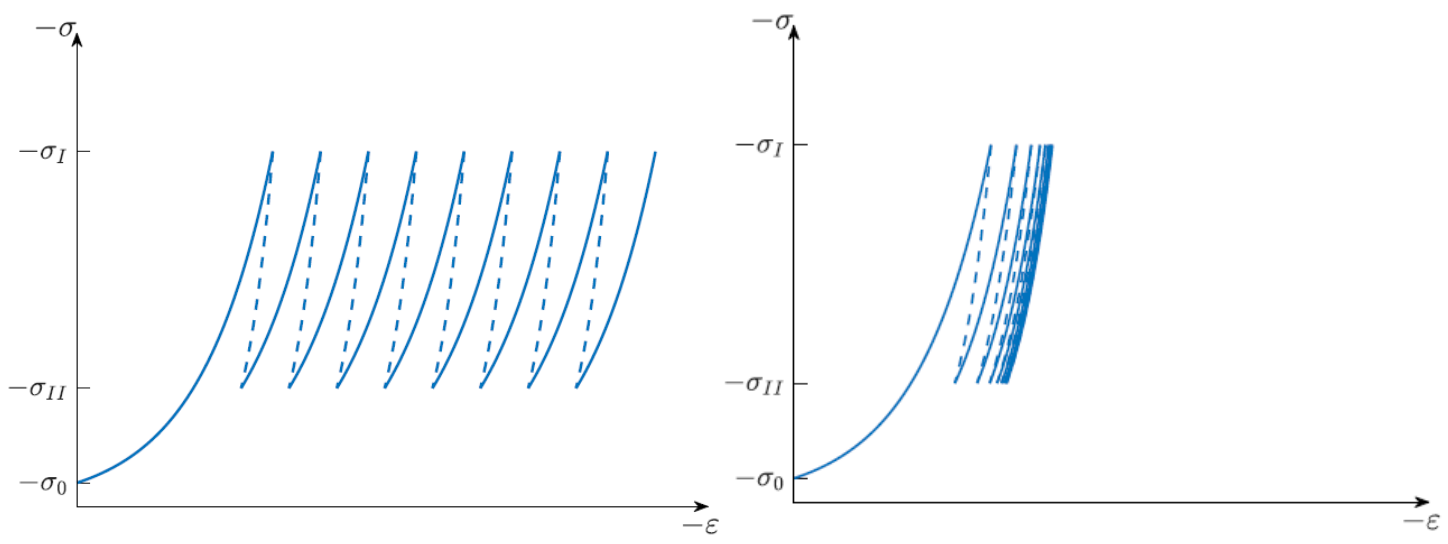

Fig. 1. Response of the constitutive equation (31) under isotropic stress cycles for the parameters $c=-550, a=0.33$ and $\sigma_{0}=-20, \sigma_{I}=-300, \sigma_{I I}=-100$; loading paths are shown by solid curves and unloading paths are shown by dashed curves. Left panel: without shakedown, $f_{\mathrm{c}}=1$. Right panel: with shakedown $f_{\mathrm{c}}=e^{-n / d}$ for $n=8$ and $d=3$

Under cyclic loading with small stress amplitudes, the model exhibits an unlimited accumulation of deformation, which is physically not relevant for granular materials. The so-called unlimited ratchetting for the case of stress cycles under isotropic condition is illustrated in Figure 1 (left panel) for the special case of $f_{\mathrm{c}}=1$. Although the model describes different incremental stiffness for loading and unloading, the amount of the incremental stiffness is only a function of the current stress and the direction of the strain rate and, consequently, independent on the number of cycles. In order to overcome this drawback different concepts for introducing appropriate state variables and memory functions depending on the loading history where proposed in enhanced versions of hypoplastic models $[8,30]$.

In order to improve the simplified hypoplastic model considered in the present paper a socalled shakedown factor $f_{\mathrm{c}}$ is added in the nonlinear part of the constitutive equation (29). To demonstrate the effect of the shakedown factor $f_{\mathrm{c}}$ on the extended equation (31) the following exponential function is introduced:

$$
f_{\mathrm{c}}=e^{-n / d}
$$

where $n$ is the number of cycles and a parameter $d>0$ triggers the amount of diminution of ratchetting. The selection of the function for factor $f_{\mathrm{c}}$ is motivated by the experimental 
observations with granular materials that the effect of ratchetting declines with the number of cycles and finally a so-called shakedown is reached. Fig. 1 (right panel) shows the shakedown behavior of Eq. (30) under isotropic stress cycles.

It is obvious that $f_{\mathrm{c}} \rightarrow 0$ in (37) for $n \rightarrow \infty$ and equation (30) reduces to the following incrementally linear constitutive relation:

$$
\frac{d \boldsymbol{\sigma}}{d t}=c\left(a^{2} \operatorname{tr}(\boldsymbol{\sigma}) \dot{\varepsilon}+\frac{\boldsymbol{\sigma}: \dot{\varepsilon}}{\operatorname{tr}(\boldsymbol{\sigma})} \boldsymbol{\sigma}\right)
$$

Equation (38) describes a nonlinear elastic material behavior and the present version belongs to the class of hypoelasticity. For alternative representations of $\mathbf{L}(\boldsymbol{\sigma}): \dot{\boldsymbol{\varepsilon}}$ also a hypereleastic material behavior can be considered. It can be noted that the feature of factor $f_{\mathrm{c}}$ in the constitutive equation (30) has certain similarities to the pressure and void space dependent density factor embedded in the hypoplastic model for cohesionless granular materials (e.g. [3] and [19]). In particular, cyclic loading leads to a rearrangement of the grains within the grain skeleton and, with an increase of the number of cycles with small stress amplitudes, the void space between the grain skeleton reduces until the minimum possible value of a given granular material is reached. Experiments with granular materials show that the shakedown behavior not only depends on the current backing density of the material but also on the amount of the stress amplitude and the direction and history of the loading path. Therefore, the simulation of general path dependent material properties requires a more sophisticated modeling.

This work was supported by the OeAD Scientific \& Technological Cooperation (WTZ CZ 18/2020: "Hysteresis in Hypo-Plastic Models") financed by the Austrian Federal Ministry of Science, Research and Economy (BMWFW) and by the Czech Ministry of Education, Youth and Sports (MŠMT). Further support by the European Research Council (ERC) under the European Union's Horizon 2020 Research and Innovation Programme, advanced grant No. 668998 OCLOC (V.A.K), by the Russian Foundation for Basic Research (RFBR), project 18-29-10007 (V.A.K), by the GA ̌́R Grant No. 20-14736S (P.K. and G.A.M.), and by the European Regional Development Fund, project No. CZ.02.1.01/0.0/0.0/16_019/0000778 (P.K.) is gratefully acknowledged.

\section{References}

[1] B.D.Annin, V.A.Kovtunenko, V.M.Sadovskii, Variational and hemivariational inequalities in mechanics of elastoplastic, granular media, and quasibrittle cracks, In: Analysis, Modelling, Optimization, and Numerical Techniques, G.O.Tost, O.Vasilieva (eds.), Springer Proc. Math. Stat., Vol. 121, 2015, 49-56.

[2] P.J.Armstrong, C.O.Frederick, A mathematical representation of the multiaxial Bauschinger effect, C.E.G.B., Report RD/B/N, Vol. 731, 1966.

[3] E.Bauer, Calibration of a comprehensive hypoplastic model for granular materials, Soils Found., 36(1996), 13-26.

[4] E.Bauer, Conditions for embedding Casagrande's critical states into hypoplasticity, Mech. Cohes.-Frict. Mat., 5(2000), 125-148.

[5] E.Bauer, Modelling limit states within the framework of hypoplasticity, In: AIP Conf. Proc., Vol. 1227, J.Goddard, P.Giovine, J.T.Jenkin (eds.), 2010, 290-305.

[6] E.Bauer, V.A.Kovtunenko, P.Krejčí, N.Krenn, L.Siváková, A.V.Zubkova, Modified model for proportional loading and unloading of hypoplastic materials, In: Extended Abstracts 
Spring 2018. Singularly Perturbed Systems, Multiscale Phenomena and Hysteresis: Theory and Applications, A.Korobeinikov, M.Caubergh, T.Lázaro, J.Sardanyés (eds.), Trends in Mathematics, Vol. 11, Birkhäuser, Ham, 2019, 201-210.

[7] E.Bauer, V.A.Kovtunenko, P.Krejčí, N.Krenn, L.Siváková. A.V.Zubkova, On proportional deformation paths in hypoplasticity, Acta Mechanica, 231(2020), 1603-1619.

DOI: $10.1007 /$ s00707-019-02597-3

[8] E.Bauer, W.Wu, A hypoplastic model for granular soils under cyclic loading, In: Modern Approaches to Plasticity, D.Kolymbas (ed.), Elsevier, 1993, 247-258.

[9] A.F.Bower, Cyclic hardening properties of hard-drawn copper and rail steel, J. Mech. Phys. Solids, 37(1989), 455-470.

[10] M.Brokate, P.Krejčí, Maximum norm wellposedness of nonlinear kinematic hardening models, Contin. Mech. Thermodyn., 9(1997), 365-380.

[11] M. Brokate, P.Krejčí, Wellposedness of kinematic hardening models in elastoplasticity, Math. Model. Num. Anal., 32(1998a), 177-209.

[12] M.Brokate, P.Krejčí, On the wellposedness of the Chaboche model, In: Control and Estimation of Distributed Parameter Systems, W.Desch, F.Kappel, K.Kunisch (eds.), Int. Series Num. Math., Vol. 26, Birkhäuser, Basel, 1998b, 67-79.

[13] D.Caillerie, R.Chambon, Existence and uniqueness for rate problems of geomechanics, Revue Française de Génie Civil, 8(2004), 537-561.

[14] J.-L.Chaboche, Constitutive equations for cyclic plasticity and cyclic viscoplasticity, Int. J. Plasticity, 5(1989), 247-302.

[15] J.-L.Chaboche, On some modifications of kinematic hardening to improve the description of ratchetting effects, Int. J. Plasticity, 7(1991), 661-678.

[16] J.-L.Chaboche, Modeling of ratchetting: evaluation of various approaches, Eur. J. Mech., A/Solids, 13(1994), 501-518.

[17] G.Duvaut, J.-L.Lions, Inequalities in Mechanics and Physics, Springer, Berlin, 1976.

[18] K.Gröger, Initial value problems for elastoplastic and elasto-viscoplastic systems, In: Nonlinear Analysis, Function Spaces and Applications, S.Fučík, A.Kufner (eds), Teubner, Leipzig, 1979, 95-127.

[19] G.Gudehus, A comprehensive constitutive equation for granular materials, Soils Found., 36(1996), 1-12.

[20] G.Gudehus, Physical Soil Mechanics, Springer, Berlin, Heidelberg, 2011.

[21] A.M.Khludnev, V.A.Kovtunenko, Analysis of Cracks in Solids, WIT-Press, Southampton, Boston, 2000.

[22] D.Kolymbas, An outline of hypoplasticity, Arch. Appl. Mech., 61(1991), 143-151.

[23] D.Kolymbas, G.Medicus, Genealogy of hypoplasticity and barodesy, Int. J. Numer. Anal. Methods Geomech., 40(2016), 2532-2550. DOI: 10.1002/nag.2546

[24] V.A.Kovtunenko, P.Krejčí, E.Bauer, L.Siváková, A.V.Zubkova, On Lyapunov stability in hypoplasticity, In: Proc. Equadiff 2017 Conference, K.Mikula, D.Ševčovič, J.Urbán (Eds.), Slovak University of Technology, Bratislava, 2017, 107-116. 
[25] V.A.Kovtunenko, P.Krejčí, N.Krenn, E.Bauer, L.Siváková, A.V.Zubkova, On feasibility of rate-independent stress paths under proportional deformations within hypoplastic constitutive model for granular materials, Mathematical Models in Engineering, 5(2019), 119-126.

[26] J.Lemaitre, J.-L.Chaboche, Mechanics of Solid Materials, Cambridge University Press, 1990.

[27] D.Mašín, Modelling of Soil Behaviour with Hypoplasticity: Another Approach to Soil Constitutive Modelling, Springer, Switzerland, 2019.

[28] E.Melan, Zur Plastizität des räumlichen Kontinuums, Ingenieur-Archiv, 9(1938), 116-126.

[29] J.Nečas, I.Hlaváček, Mathematical Theory of Elastic and Elastoplastic Bodies: An Introduction, Elsevier, Amsterdam, 1981.

[30] A.Niemunis, I.Herle, Hypoplastic model for cohesionless soils with elastic strain range, Mech. Cohes.-Frict. Mat., 2(1997), 279-299.

[31] W.Prager, Recent developments in the mathematical theory of plasticity, J. Appl. Phys., 20(1949), 235-241.

[32] L.Prandtl, Ein Gedankenmodell zur kinetischen Theorie der festen Körper, Z. Angew. Math. Mech., 8(1928), 85-106.

[33] K.R.Rajagopal, A.R.Srinivasa, On a class of non-dissipative materials that are not hyperelastic, Proc. R. Soc. A., 465(2009), 493-500.

[34] O.Sadovskaya, V.Sadovskii, Mathematical Modeling in Mechanics of Granular Materials, Springer, Berlin, Heidelberg, 2012.

[35] C.Truesdell, Remarks on hypo-elasticity, J. Res. Natl. Bur. Stand., Ser. B. Math. Math. Phys., 67B(1963), 141-143.

\title{
Циклическое поведение упрощенной модели гипопластичности и пластичности с нелинейным кинематическим упрочнением
}

\author{
Виктор А. Ковтуненко \\ Университет Грац \\ Грац, Австрия \\ Институт гидродинамики им. М. А. Лаврентьева СО РАН \\ Новосибирск, Российская Федерация \\ Эрих Бауер \\ Технический университет Грац \\ Грац, Австрия \\ Ян Элиаш \\ Университет Грац \\ Грац, Австрия \\ Павел Крейчи \\ Технический университет в Праге \\ Прага, Чешская Республика \\ Жизель А. Монтейро \\ Институт математики Чешской академии наук \\ Прага, Чешская Республика \\ Ленка Стракова (Сивакова) \\ Технический университет в Праге \\ Прага, Чешская Республика
}


Аннотация. Статья описывает вопросы моделирования и математического анализа корректности задач, мотивированных циклическим поведением уравнений состояния, которые не зависят от скорости, в рамках теорий гипопластичности и пластичности с нелинейным кинематическим упрочнением. По сравнению с классической теорией упругопластичности в гипопластичности нет необходимости разлагать деформацию на упругую и пластичную части. Два рассматриваемых типа нелинейных подходов показывают некоторое сходство в структуре определяющих соотношений, которое важно для описания поведения пластических материалов. При этом обе модели демонстрируют неограниченные флуктуации при циклической нагрузке. В результате численного моделирования продемонстрировано, как ограничение флуктуаций может быть достигнуто с помощью слегка улучшенного простого гипопластического уравнения, предложенного Бауэром.

Ключевые слова: теория пластичности, гипопластичность, система, не зависящая от скорости, гистерезис, циклическая нагрузка, математическая корректность, численное моделирование. 